\title{
Correction to: SPECT/CT imaging of apoptosis in aortic aneurysm with radiolabeled duramycin
}

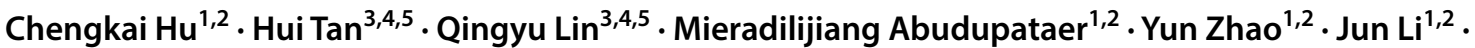 \\ Jiawei $\mathrm{Gu}^{1,2} \cdot$ Dengfeng Cheng ${ }^{3,4,5} \cdot$ Chunsheng Wang ${ }^{1,2} \cdot$ Kai Zhu $^{1,2} \cdot$ Hao Lai $^{1,2}$
}

Published online: 17 August 2019

๑) Springer Science+Business Media, LLC, part of Springer Nature 2019

\section{Correction to: Apoptosis}

https://doi.org/10.1007/s10495-019-01554-8

The original version of this article unfortunately contains errors in Fig. 4.

An incorrect Fig. $4 \mathrm{~d}$ is published which is actually a repetition of Fig. 2c (i.e., apoptosis rate in control vs. H2O2treated group). The correct Fig. $4 \mathrm{~d}$ should be the aortic diameter of control vs. experimental groups.

Chengkai Hu and Hui Tan have contributed equally to this work.

The original article can be found online at https://doi.org/10.1007/ s10495-019-01554-8.

Dengfeng Cheng

cheng.dengfeng@zs-hospital.sh.cn

$\triangle$ Kai Zhu

zhu.kai1@zs-hospital.sh.cn

$\triangle$ Hao Lai

lai.hao@zs-hospital.sh.cn

1 Department of Cardiac Surgery, Zhongshan Hospital, Fudan University, 1609 Xietu Road, Shanghai 200032, China

2 Shanghai Institute of Cardiovascular Diseases, Shanghai 200032, China

3 Department of Nuclear Medicine, Zhongshan Hospital, Fudan University, 180 Fenglin Road, Shanghai 200032, China

4 Institute of Nuclear Medicine, Fudan University, Shanghai 200032, China

5 Shanghai Institute of Medical Imaging, Shanghai 200032, China
Also, the order of part figures (alblcld) in Fig. 4e is incorrect.

The correct Fig. 4 is given below. 

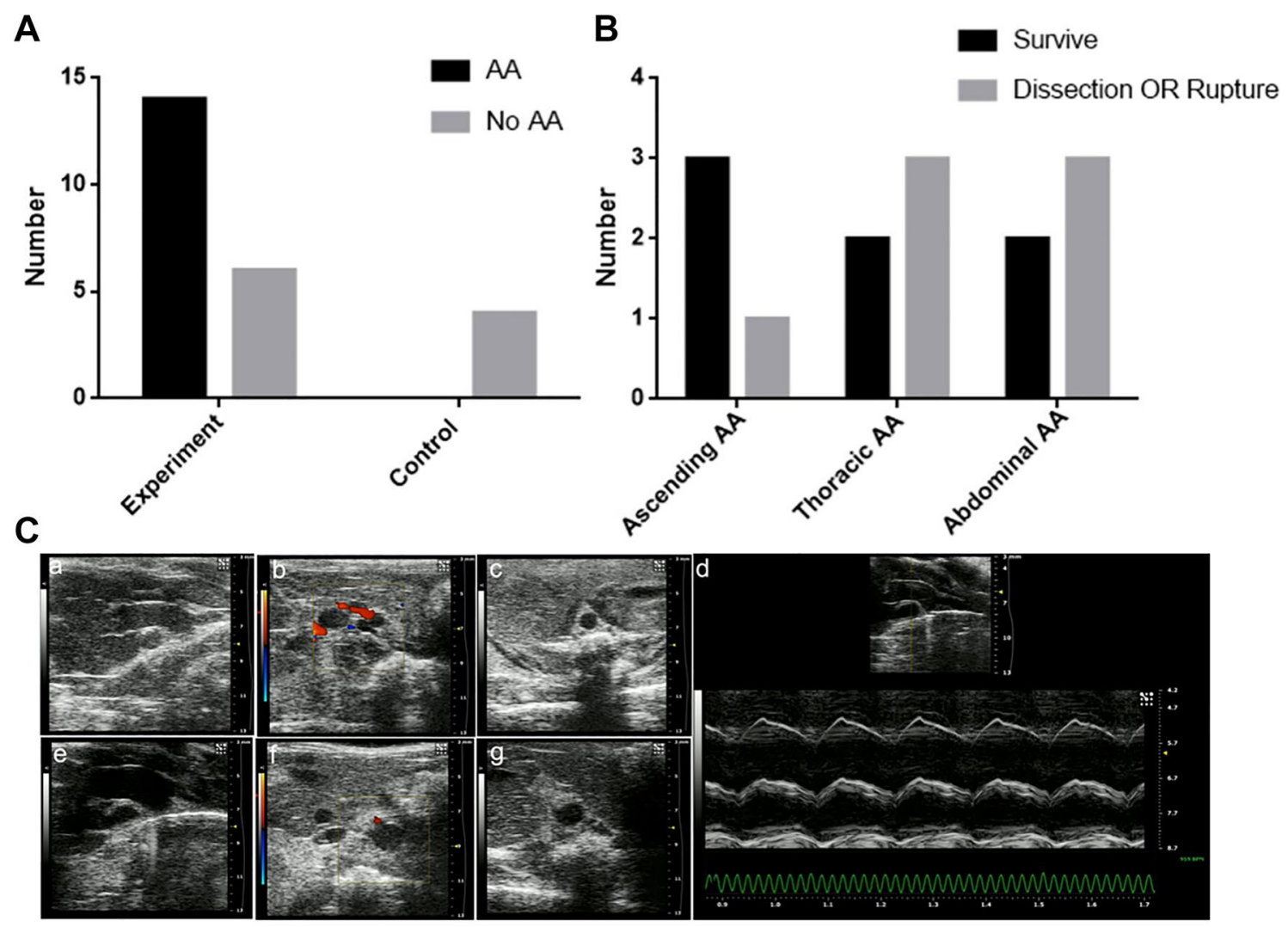

D

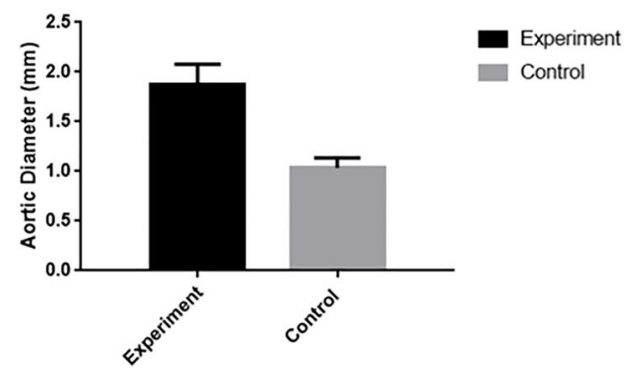

Fig. 4 Aortic aneurysm formation in mice using BAPN and angiotensin-II. a The incidence of AA formation was significantly different between the experimental and control groups. The overall AA incidence was $70 \%(14 / 20)$. b The types of AA in the experimental group were ascending AA (4/14), TAA (5/14), and AAA (5/14). The survival rate of patients with AAs was 75,40 , and $40 \%$ in the three groups, respectively. C Diameters of the ascending and abdominal
E

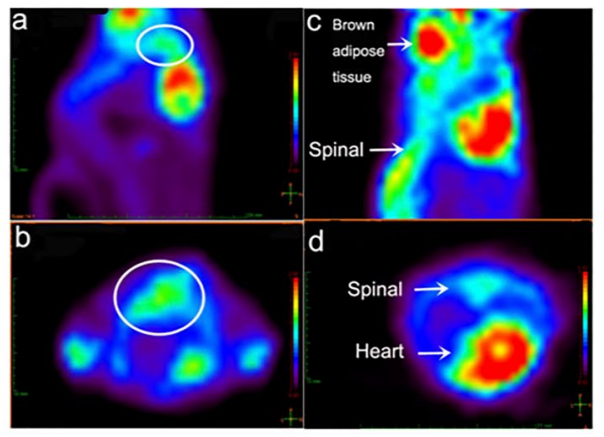

Publisher's Note Springer Nature remains neutral with regard to jurisdictional claims in published maps and institutional affiliations.

aorta were larger in the experimental group (e-g) compared with the control group $(\mathrm{a}-\mathrm{c})$. M-mode echocardiography in AAs is shown in d. $\mathbf{d}$ The aortic diameter was visibly larger in the experimental group compared with the control group $(1.8 \pm 0.2 \mathrm{~mm}$ vs. $1.0 \pm 0.1 \mathrm{~mm}$, $P<0.01$ ). $\mathrm{e}^{18} \mathrm{~F}-\mathrm{FDG}$ uptake in AA (a and b) AA is higher than in the control group (c and $\mathrm{d}$ ). The white circle indicates positive imaging 